\title{
The axial anomaly and the phases of dense QCD
}

\author{
Gordon Baym, ${ }^{1}$ Tetsuo Hatsuda, ${ }^{2}$ Motoi Tachibana, ${ }^{3}$ and Naoki \\ Yamamoto $^{2}$ \\ ${ }^{1}$ Department of Physics, University of Illinois, 1110 W. Green St., Urbana, Illinois \\ 61801 \\ ${ }^{2}$ Department of Physics, The University of Tokyo, Tokyo 113-0033, Japan \\ ${ }^{3}$ Department of Physics, Saga University, Saga 840-8502, Japan \\ E-mail: gbaym@uiuc.edu
}

\begin{abstract}
.
The QCD axial anomaly, by coupling the chiral condensate and BCS pairing fields of quarks in dense matter, leads to a new critical point in the QCD phase diagram 1, 2, which at sufficiently low temperature should terminate the line of phase transitions between chirally broken hadronic matter and color superconducting quark matter. The critical point indicates that matter at low temperature should cross over smoothly from the hadronic to the quark phase, as suggested earlier on the basis of symmetry. We review here the arguments, based on a general Ginzburg-Landau effective Lagrangian, for the existence of the new critical point, as well as discuss possible connections between the QCD phase structure and the BEC-BCS crossover in ultracold trapped atomic fermion systems at unitarity. and implications for the presence of quark matter in neutron stars.
\end{abstract}

PACS numbers: 12.38.-t,12.38.Mh,26.60.+c 


\section{Introduction}

Understanding the properties of matter under extreme conditions has been an important motivation for carrying out ultrarelativistic heavy ion collisions [3]. The general features of dense matter can be summarized in the QCD phase diagram, various aspects of which have been discussed by several speakers here, e.g., [4-7]. Crucial refinements of the phase diagram have been the high temperature critical point - point C in Fig. 1 - (reviewed in [6]), and the phases of color superconducting quark matter at low temperature and high density [8].

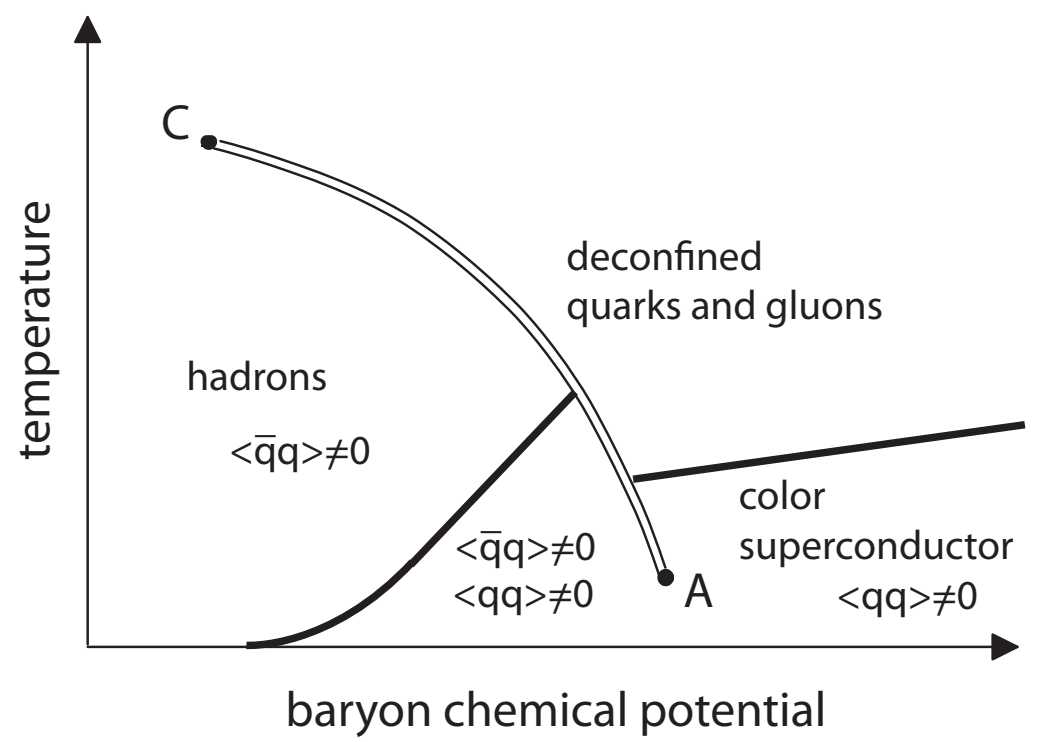

Figure 1. Schematic phase diagram of dense QCD with two light up and down quarks and a medium heavy strange quark, showing the new critical point, A, at low temperature, and regions of broken chiral symmetry, $\langle\bar{q} q\rangle \neq 0$, and regions of diquark pairing, $\langle q q\rangle \neq 0$. In the region to the left of A broken chiral symmetry and quark pairing coexist.

Here we discuss how the $\mathrm{U}(1)_{A}$ axial anomaly, by coupling two competing phenomena - quark-anti-quark pairing, characterized by a chiral condensate $\Phi \sim\langle\bar{q} q\rangle$, and quark-quark pairing, characterized by a diquark condensate $d \sim\langle q q\rangle$-induces a second critical point in the phase diagram, point A in Fig. 1. The anomaly leads to an effective six quark 't Hooft interaction, $\sim \operatorname{det}_{i j}\left\langle\bar{q}_{R}^{j} q_{L}^{i}\right\rangle$, where $i$ and $j$ are flavor indices, and $R$ and $L$ denote quark chiralities; this interaction in turn implies couplings $\sim \Phi^{3}$ and $\Phi d^{\dagger} d$; the latter coupling, which favors coexistence of chiral symmetry breaking and BCS quark pairing, is responsible for the new critical point. 


\section{Ginzburg-Landau effective Lagrangian}

Since in the neighborhood of chiral symmetry breaking and quark pairing transitions $d$ and $\Phi$ are small, we construct the free energy by means of a model-independent Ginzburg-Landau effective Lagrangian developed in powers of $d$ and $\Phi$. Owing to space limitations, we make discuss only the simple case that for three massless flavors, $\Phi=\operatorname{diag}(\sigma, \sigma, \sigma)$, and the pairing field is in the color-flavor locked phase [8], $d_{L}=$ $-d_{R}=\operatorname{diag}(d, d, d)$, with real order parameters $\sigma$ and $d$. The minus sign corresponds to the vacuum having even parity. See Refs. 1] and 2] for full details. The free energy, measured with respect to that for $\sigma=d=0$, is a sum, $\Omega_{3 F}=\Omega_{0}+\Omega_{\chi}+\Omega_{d}+\Omega_{\chi d}$, of contributions from the chiral field, the pairing field, and their interaction, respectively. The chiral term has the form [9],

$$
\Omega_{\chi}=\frac{a}{2} \sigma^{2}-\frac{c}{3} \sigma^{3}+\frac{b}{4} \sigma^{4}+\frac{f}{6} \sigma^{6} ;
$$

the $c$ term arises from the axial anomaly. The system breaks chiral symmetry when $a<2 c^{2} / 9 b$, for $b>0$. The $f \sigma^{6} / 6$ is needed to stabilize the system should $b$ be negative. The pairing free energy [10] is

$$
\Omega_{d}=\frac{\alpha}{2} d^{2}+\frac{\beta}{4} d^{4} ;
$$

the system pairs when $\alpha<0$. Finally, the interaction between the fields, to fourth order in the fields, is

$$
\Omega_{\chi d}=-\gamma d^{2} \sigma+\lambda d^{2} \sigma^{2} .
$$

The $\gamma$ term, which breaks the $U(1)_{A}$ symmetry down to $Z(6)$, originates from the axial anomaly $₫$ The 't Hooft interaction implies that the coefficient $c$ of the $\sigma^{3}$ term, and $\gamma$ have the same (positive) sign and similar magnitude. The term $\gamma$ term encourages the coexistence of the pairing and chiral fields, and is eventually responsible for the new critical point; a non-zero $d^{2}$ acts as external field for $\sigma$, washing out the first order transition for large $d^{2}$, as in a magnetic system in external field. On the other hand, in the presence of the $\gamma$ term, the $\lambda d^{2} \sigma^{2}$ plays little quantitative role, since $\lambda / \beta$ is expected to be $\ll 1[1]$.

The phase structure for three massless flavors in the $a, \alpha$ plane implied by $\Omega_{3 F}=$ $\Omega_{0}+\Omega_{\chi}+\Omega_{d}+\Omega_{\chi d}$, for $b>0$, is shown in the left panel of Fig. 2. The "normal" (NambuGoldstone like) region to the upper left has broken chiral symmetry, while in the "QGP" region in the upper right, chiral symmetry is restored; in neither of these regions are the quarks paired. The point $\mathrm{D}$ is always at positive $\alpha$, since a non-zero $\sigma$ enhances the pairing gap, via the $-\gamma d^{2} \sigma$ in Eq. (3), and $\sigma$ is larger at smaller chemical potential [1, 2]. The region to the lower right is color superconducting. As a consequence of the $\gamma$ coupling, the double line (which for $\gamma=0$ runs parallel to the $\alpha$ axis down to $-\infty$ ) terminates at a critical point - always at positive $a>0$ and negative $\alpha$ - connecting

$\ddagger$ The general Ginzburg-Landau Lagrangian [1, 2], aside from the two terms arising from the anomaly, is constructed to be fully invariant under the group $\mathcal{G}=\mathrm{SU}(3)_{\mathrm{L}} \times \mathrm{SU}(3)_{\mathrm{R}} \times \mathrm{U}(1)_{\mathrm{B}} \times \mathrm{U}(1)_{\mathrm{A}} \times \mathrm{SU}(3)_{\mathrm{C}}$. 
the color superconducting region in the lower right continuously to a coexistence region to the left. The coexistence region is characterized by both chiral symmetry breaking and pairing. As a consequence of the critical point, the color superconducting region also has a non-zero chiral field, $\sigma$. Across phase transition marked by the double line, $\sigma$ decreases discontinuously with increasing $a$.

Similarly for two massless flavors $\left(m_{s}=\infty\right)$, we write $\Phi=\operatorname{diag}(\sigma, \sigma, 0)$ and $d_{L}=-d_{R}=\operatorname{diag}(0,0, d)$, corresponding to the $2 \mathrm{SC}$ state of color superconductivity. With this color-flavor structure, the cubic terms in $\sigma$ and $d$ are identically zero, leading to a phase diagram with a much simpler structure than for three massless flavors. The free energy is

$$
\Omega_{2 F}=\left(\frac{a}{2} \sigma^{2}+\frac{b}{4} \sigma^{4}+\frac{f}{6} \sigma^{6}\right)+\left(\frac{\alpha}{2} d^{2}+\frac{\beta}{4} d^{4}\right)+\lambda d^{2} \sigma^{2},
$$

where the coefficients are the same as in the three flavor case times numerical factors of order unity.

The right panel of Fig. 2 shows the phase structure for two massless flavors in the $a, \alpha$ plane, for $b>0$. The solid lines are second order transitions. Unlike for three flavors, there is no critical point; rather in the color superconducting phase, $\sigma=0$, owing to the absence of the $\gamma d^{2} \sigma$ coupling.

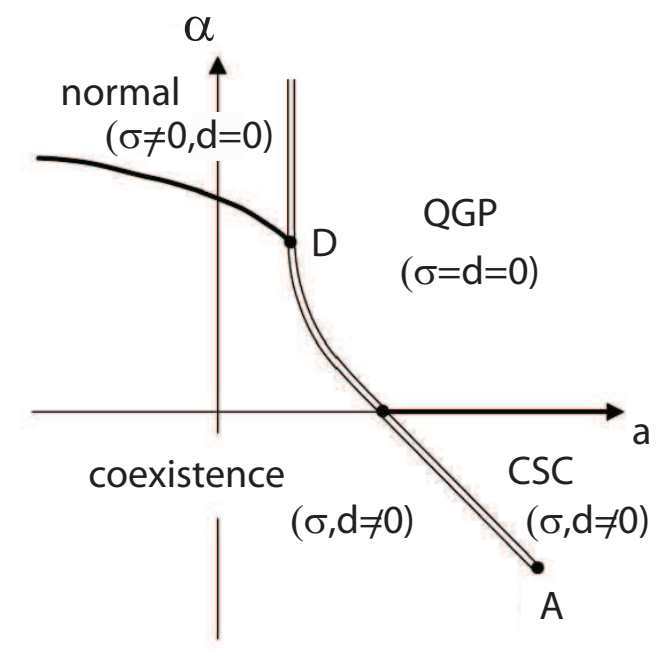

3 FLAVORS

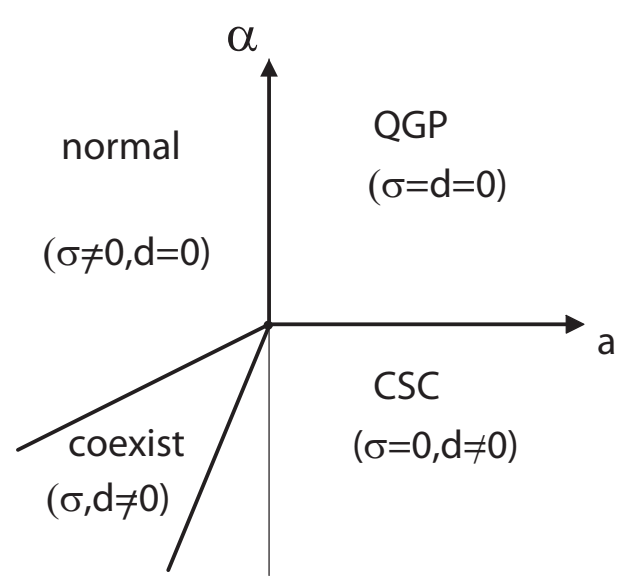

2 FLAVORS

Figure 2. The phase structure in the $a, \alpha$ plane for three massless flavors (left panel) and two massless flavors (right panel). The critical point for three massless flavors is denoted by A.

In the presence of the axial anomaly, the coexistence and the color superconducting phases have same symmetry, allowing a crossover between them. On the other hand, 
the normal and coexistence phases realize $\mathrm{U}(1)_{\mathrm{B}}$ differently and the boundary between these regions, the solid line in both panels of Fig. 2, is sharp.

Mapping the phase diagram from the $a, \alpha$ plane to the baryon chemical potential - temperature plane requires a dynamical picture to calculate, for realistic quark masses, the Ginzburg-Landau parameters, and to treat the full interplay between chiral symmetry breaking, quark pairing, and confinement, e.g., via lattice simulations or effective theories of confinement [11]. Generally, we expect $a$ and $\alpha$ to decrease from positive to negative with decreasing temperature. We further expect the situation with realistic quark masses to be intermediate between the two and three flavor massless cases, and thus expect the schematic phase diagram for realistic quark masses shown in Fig. 1. NJL estimates of the Ginzburg-Landau parameters [2] indicate that the critical values of $a$ and $\alpha$ at the critical point $\mathrm{A}$ are reached at finite temperature, so that the critical point occurs at non-zero temperature. With increasing strange quark mass, the critical point A is expected to move to lower temperature. The solid line on the left demarcating the normal and coexistence regions must touch the double line at higher temperature than the color superconducting region. The new critical point is at temperature below the critical temperature for onset of color pairing, and thus would be difficult to study at RHIC and LHC; however, it may be accessible at FAIR.

The three flavor coexistence region to the left in which both $d$ and $\sigma$ are nonzero is an intermediate state between color-paired quark matter and hadronic matter. The details of this state, which depend crucially on understanding interplay with confinement, remain to be delineated; below we discuss suggestive similarities of this structure to the BEC-BCS crossover in ultracold atomic systems. An essential feature of the schematic phase diagram here is the continuity between the coexistence region and the color-paired region. The Ginzburg-Landau picture points towards an explicit realization of "hadron-quark continuity" [12].

\section{BEC-BCS crossover and the deconfinement transition}

Although the energy scales in quark-gluon plasmas are some 21 orders of magnitude greater than in ultracold trapped atomic systems (on the sub-microkelvin scale), strongly interacting atomic fermion systems share certain intriguing overlaps with quark-gluon plasmas, e.g., first viscosities approaching the KSS bound [13], and possible BCS pairing in imbalanced fermionic gases [14]. Particular relevant, the crossover from the coexistence region to quarks at finite baryon density, Fig. 1, and the crossover from BoseEinstein condensation of molecules to BCS-paired superfluids in two component cold fermionic gases, Fig. 3, bear suggestive similarities, opening possible new approaches to the deconfinement transition.

Figure 3 shows the finite temperature phase diagram of a gas of two equally populated hyperfine states of ultracold fermionic atoms. The atoms interact with an attractive potential, with an s-wave scattering length, $a$, which can be tuned in experiment from large positive to large negative values by means of a Feshbach resonance 


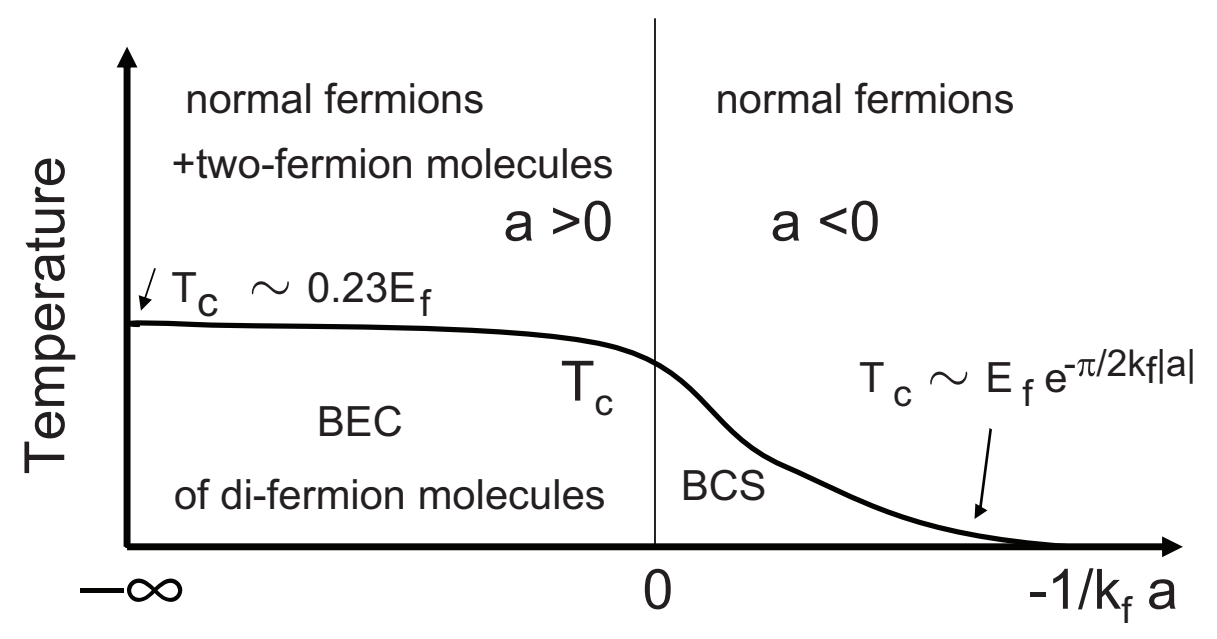

Figure 3. Phase diagram of ultracold atomic fermions as a function of the negative of the inverse scattering length $a$, in units of the Fermi momentum. The continuous curve is the transition temperature for condensation, approaching the weakly interacting Bose-Einstein transition temperature to the left and the BCS transition temperature to the right; $E_{f}$ is the free particle Fermi energy. The transition between the BEC and BCS regions is a smooth crossover.

in an external magnetic field. The horizontal axis is $-1 / k_{f} a$, where $k_{f}$ is the Fermi momentum of the gas. At small positive $a$, the system has strongly bound states - difermion molecules - which undergo Bose condensation at low temperature, while at small negative $a$ the fermions become BCS paired at low temperatures. Through the Feshbach resonance, where $|a|$ becomes large compared with the interparticle spacing, the strongly interacting system is scale free, similar to a quark-gluon plasma at high temperature. Remarkably, in the region between these two extremes the system undergoes a gradual crossover from a BEC superfluid of molecules to a BCS paired superfluid [15]. Nothing dramatic happens as one goes through the limit $|a| \rightarrow \infty$; rather, the molecules continuously expand in size from tightly bound on the BEC side to widely spaced pairs in the BCS regime, while the system remains superfluid [16].

The correspondences with the phase diagram of dense QCD matter, Fig. 1, are apparent. At very large baryon chemical potential, $\mu$, and low temperature the matter is BCS-paired, as are the atomic systems at large $-1 / k_{f} a$, and low temperature. As the temperature increases the atomic system becomes a fluid of normal unpaired fermions, while the QCD system becomes an unpaired plasma. Furthermore, with decreasing $-1 / k_{f} a$ the atomic system continuously transforms into a Bose-Einstein condensate of di-fermion molecules; similarly QCD matter at low temperature goes from the colorpaired states at large $\mu$ smoothly under the critical point, to the coexistence state in which both $\langle q q\rangle$ and $\langle\bar{q} q\rangle$ are non-zero. 
Were the color symmetry group only $\mathrm{SU}(2)$, one could make a strong analogy between the atomic and quark systems - diquark bosonic baryons would go over into BCS pairs at higher densities, just as the atomic molecules expand into BCS pairs through the Feshbach resonance. However in $\mathrm{SU}(3)_{\mathrm{C}}$, the system must develop strong three-quark correlations at low densities from the two-quark correlations at high densities. The atomic systems suggest that these correlations can enter through (2SC) BCS pairs shrinking into diquarks as the system enters the coexistence region, forming a Bose-Einstein condensate of diquarks (the extension of the 2SC phase); and that at the solid line in Fig. 1, the diquarks bind to the unpaired quarks to form nucleons. However, construction of such a picture requires understanding more fully the effect of residual interactions on the momentum distributions of the quarks [17].

\section{Dense quark matter and neutrons stars}

The canonical phenomenological approach to deduce the onset of quark matter in high density nuclear matter is to calculate the equations of state of hadronic matter and quark matter; when the thermodynamic potential for quark matter becomes lower than that for hadronic matter, the system is presumed to undergo a first order phase transition to the quark state, e.g., [18]. Typical nuclear matter equations of state are based on nucleon-nucleon interactions deduced from scattering experiment, together with three body forces constrained by the light nuclei, e.g., [19]. From such an approach one would conclude that quark matter first appears at baryon densities, $n$, of order 7-10 times nuclear matter density, $n_{0}$, and thus the observation of high mass neutron stars (e.g., Vela X-1, Cyg X-2, where $M$ could be as large as $1.8 M_{\odot}$ ) would practically preclude the existence of quark matter cores in neutron stars.

However, an equation of state based on nucleon interactions alone, while accurately describing neutron star matter in the neighborhood of nuclear matter density, has fundamental limitations. Beyond a few times $n_{0}$ one should not expect that the forces between particles can be described in terms of static few-body potentials; since the characteristic range of the nuclear forces is $\sim 1 / 2 m_{\pi}$, the parameter measuring the relative importance of three and higher body forces is of order $n /\left(2 m_{\pi}\right)^{3} \sim 0.4 n / \mathrm{fm}^{-3}$, so that at densities well above $n_{0}$ a well defined expansion in terms of two-, three-, or more, body forces no longer exists. The nucleonic equation of state furthermore does not take into account the rich variety of hadronic degrees of freedom that enter with increasing density. Nor can one continue to assume that the system at higher densities can even be described in terms of well-defined "asymptotic" laboratory particles.

More realistically, one should expect a gradual onset of quark degrees of freedom in dense matter, degrees of freedom not accounted for by nucleons interacting via static potentials. Indeed once nucleons overlap considerably the matter should percolate, opening the possibility of their quark constituents propagating throughout the system (although near the onset of percolation valence quarks may prefer to remain bound in triplets, mimicking nucleons, leaving the matter a color insulator) [20, 21]. Furthermore, 
the transition from hadronic to quark matter at low temperature, as suggested by Fig. 1, would involve the matter entering the coexistence phase, possibly a diquark Bose-Einstein condensate, and then smoothly crossing over to superfluid quark matter. The relation of the transition to the coexistence phase and percolation remains an open question. A firm assessment of the role of quarks in neutron stars must await a better understanding of mechanism of quark deconfinement with increasing baryon density.

\section{Acknowledgments}

This research was supported in part by the Grants-in-Aid of the Japanese Ministry of

Education, Culture, Sports, Science, and Technology (No. 18540253), and by U.S. NSF Grants No. PHY03-55014 and PHY07-01611. Author GB thanks the University of Tokyo for its kind hospitality as well as support through the COE.

\section{References}

[1] T. Hatsuda, M. Tachibana, N. Yamamoto, and G. Baym, Phys. Rev. Lett. 97, 122001 (2006).

[2] N. Yamamoto, M. Tachibana, T. Hatsuda, and G. Baym, Phys. Rev. D 76, 074001 (2007).

[3] G. Baym, Nucl. Phys. A698, xxiii (2001).

[4] L. McLerran, this volume.

[5] S. Gupta. this volume.

[6] M.P. Lombardo, this volume.

[7] K. Fukushima, this volume.

[8] M.G. Alford, K. Rajagopal, T. Schaefer, and A. Schmitt, Rev. Mod. Phys. (in press, 2008).

[9] R.D. Pisarski and F. Wilczek, Phys. Rev. D29, 338 (1984).

[10] K. Iida and G. Baym, Phys. Rev. D 63, 074018 (2001); ibid. 66, 059903(E) (2002).

[11] R. Pisarski, Phys. Rev. D 62 (2000); K. Fukushima, Phys. Lett. B591 (2004); S. Rößner, C. Ratti, and W. Weise Phys. Rev. D 75, 034007 (2007).

[12] T. Schäfer and F. Wilczek, Phys. Rev. Lett. 82, 3956 (1999).

[13] P.K. Kovtun, D.T. Son, and A. O. Starinets, Phys. Rev. Lett. 94, 111601 (2005); G. Rupak and T. Schaefer, Phys. Rev. A 76, 053607 (2007); T. Schaefer, Phys. Rev. A 76, 063618 (2007); G.M. Bruun and H. Smith, Phys. Rev. A 75, 043612 (2007).

[14] G. B. Partridge, W. Li, Y. A. Liao, R. G. Hulet, M. Haque, and H.T.C. Stoof, Phys. Rev. Lett. 97, 190407 (2006); C. H. Schunck, Y. Shin, A. Schirotzek, M. W. Zwierlein, and W. Ketterle, Science 316, 867 (2007).; Y. Shin, C.H. Schunck, A. Schirotzek, and W. Ketterle, arXiv:0709.3027.

[15] D.M. Eagles, Phys. Rev. 186, 456 (1969); A.J. Leggett, J. Phys. (Paris) C7, 19 (1980); P. Nozires and S. Schmitt-Rink, J. Low Temp. Phys. 59, 195 (1985).

[16] M. W. Zwierlein, J. R. Abo-Shaeer, A. Schirotzek, C. H. Schunck, and W. Ketterle, Nature 435, $1051(2005)$.

[17] H. Abuki, T. Hatsuda, and K. Itakura, Phys. Rev. D 65, 074014 (2002); K. Maeda, T. Hatsuda, and G. Baym, to be published.

[18] G. Baym and S. A. Chin, Phys. Lett. B 62, 241 (1976).

[19] A. Akmal, V.R. Pandharipande, and D.G. Ravenhall, Phys. Rev. C 58, 1804 (1998).

[20] G. Baym, Physica 96A, 131 (1979).

[21] H. Satz, Rep. Prog. Phys. 63, 1511 (2000). 